\title{
Study of the welding gas influence on a controlled short-arc GMAW process by optical emission spectroscopy
}

\author{
G. Wilhelm ${ }^{1}$, G. Gött ${ }^{2}$, H. Schöpp ${ }^{2}$, D. Uhrlandt ${ }^{2}$ \\ ${ }^{1}$ Linde AG, Unterschleissheim 85716, Germany \\ ${ }^{2}$ Leibniz-Institute for Plasma Science and Technology e.V., Felix-Haussdorf-Str. 2, \\ Greifswald 17489, Germany \\ E-mail: uhrlandt@inp-greifswald.de
}

\begin{abstract}
The controlled short arc processes, variants of the Gas Metal Arc Welding (GMAW) process, which have recently been developed, are used to reduce the heat input into the workpiece. Such a process with a wire feeding speed which varies periodically, using a steel wire and a steel workpiece to produce bead on plate welds has been investigated. As welding gases $\mathrm{CO}_{2}$ and a mixture of $\mathrm{Ar}$ and $\mathrm{O}_{2}$ have been used. Depending on the gas the properties of the plasma change, and as a consequence the weldseams themselves also differ distinctly. Optical emission spectroscopy has been applied to analyse the plasma. The radial profiles of the emission coefficients of an iron line and an argon line or an atomic oxygen line, respectively, have been determined. These profiles indicate the establishment of a metal vapour arc core which has a broader profile under $\mathrm{CO}_{2}$ but is more focussed in the centre for argon. The measured iron line emission was near to its norm maximum in case of $\mathrm{CO}_{2}$. From this fact, temperatures around $8000 \mathrm{~K}$ and a metal vapour molar fraction above $75 \%$ in the arc centre could be roughly estimated for this case. Estimations of the electrical conductivity and the arc field indicate that the current path must include not only the metal vapour arc core but also outer hot regions dominated by welding gas properties in case of argon.
\end{abstract}

PACS numbers: $52.80 . \mathrm{Mg}, 52.75 . \mathrm{Rx}, 52.70 . \mathrm{Kz}$

Keywords: gas metal arc welding, optical emission spectroscopy

Submitted to: J. Phys. D: Appl. Phys. , revised version June 10, 2010

\section{Introduction}

The standard short circuiting metal transfer is a mode of metal transfer, used for Gas Metal Arc Welding (GMAW) of metal work pieces, whereby a continuously fed wire electrode is deposited during repeated electrical short circuits in a frequency range of 20 to $150 \mathrm{~Hz}$ [1]. The transfer of the molten edge of the wire occurs during each shorting

phase of one cycle. This mode of metal transfer typically supports the use of small wire diameters in a range of 0.8 to $1.2 \mathrm{~mm}$. This welding technique is particularly useful for 
joining thin materials in any position. For thicker materials it is used to weld the root pass (first bead of a multipass weld) as well as other layers in the vertical and overhead position. The low heat input that goes along with this process is also beneficial to achieve minimum distortion of the workpiece. In general $\mathrm{CO}_{2}$ or an argon rich mixture including $\mathrm{CO}_{2}$ or $\mathrm{O}_{2}$ is used as a welding gas to run this process. The influence of different welding gases on the establishment of the arc and, hence, on the process is the aim of the present paper. Before explaining this focus in more detail, principles of the standard short circuiting metal transfer as well as of the cold metal transfer process should be shortly sketched in the following.

Figure 1 shows a typical current-voltage vs. time sketch of short circuiting transfer comprising significant points A to $\mathrm{E}$ which are explained in the following. Between points $\mathrm{A}$ and $\mathrm{C}$ the wire electrode has physical contact with the molten pool. The voltage goes down to a level near zero and the current starts to increase. The rate of the current rise is determined by the inductance of the circuit. During phase B the voltage begins to climb very slowly and the current increases towards its top level. The contact of the electrode with the weld pool is reduced to a small bridge of molten metal called jumper. Consequently, the radial magnetic force acting on the jumper (pinch effect) raises towards its maximum, too. At point $\mathrm{C}$ the jumper is just about to break through and the droplet starts being transferred into the molten pool. At point $\mathrm{D}$ the jumper is broken through and a metal vapour plasma occurs. After a few microseconds a gas-dominated welding arc is established. Phase $\mathrm{E}$ is the arc extinction phase of the cycle. The wire electrode is, once again, going to have contact with the weld pool.

The problem of the standard short circuiting metal transfer with a predetermined inductance is the spatter generation that goes along with the break through of the jumper. This is illustrated in Figure 2.

In order to minimise spatter it is necessary to control the short circuiting process. Thanks to further developments of power electronics, modern welding power sources offer the possibility to reduce the welding current immediately after the break through of the jumper by varying the inductance and generating a countervoltage while feeding the wire electrode continuously. Apart from the possibilty to optimise the process by controlling the electrical parameters, the cold metal transfer (CMT) process [2, 3] implements the wire speed as an additional parameter in its controlling system as shown for a typical process in figure 3. During the arc burning phase (phase 1) the wire electrode is fed towards the molten pool. As soon as the wire electrode contacts the weld pool the current is reduced (point 2). During the short circuit phase (phase 3) the feeding direction of the wire is changed in order to support a smooth droplet detachment, i.e., the wire moves away from the weld pool. At point 4 the jumper has just broken, a metal vapour plasma occurs and a steep current rise is initiated. When the gas dominated welding arc is established (phase 5) the feeding direction of the wire is changed once again so that the wire is moving towards the weld pool.

The heat input into the work piece during the arc burning phase is also influenced by the used welding gas. This is already known from the comparison of microsections of 
different weld seams. However, for a purposeful choice of the welding gas the physical mechanisms of their impact have to be revealed in more detail.

Optical emission spectroscopy (OES) has been proven as a powerful diagnostic method for the study of arc plasmas. Measurements of the line radiation with sufficient resolution and accuracy allow the determination of the plasma properties by several methods[4]. However, only few studies of GMAW processes by spectroscopic methods can be found in the literature $[5,6,7,8,9]$. Reasons are the more rapid variation in time, the lower reproducibility and the more complex gas mixture resulting from the metal evaporation during the process in comparison with e.g. tungsten inert gas (TIG) welding which has been studied more intensively. The presence and often considerable impact of metal vapour have been evaluated in the studies of the GMAW processes. The optical monitoring of the metal radiation can be used as a sensitive online control of the process (see e.g. [7, 8]). It has been shown in addition that the metal vapour is often concentrated in a small central region of the arc $[5,9]$.

Corresponding to the observed impact of welding gases on the weld seams in case of the CMT process, differences in the radial arc structure can be expected. Although this impact can be properly studied by OES along the arc cross section, such comparisons have been made up to now for few examples of GMAW processes only. The different radial arc structure in front of the work piece for welding gases argon and mixtures of argon and $\mathrm{CO}_{2}$ as well as the transition from a spray arc to a globular arc operation have been analysed in [9].

In the present work the arc plasma in the high-current phase of the CMT process is investigated. To reveal the different impact of inert or molecular gases as the welding gas, the CMT process with steel wire and workpiece is considered for the gases $\mathrm{CO}_{2}$ and a mixture of argon and $\mathrm{O}_{2}$. By means of the radial emission profiles of the metal vapour and the welding gas the contribution of the metal vapour to the arc operation is analysed. In addition, conclusions to the profile of the heat transfer to the workpiece and the related weld pool behaviour are drawn. The paper is organized as follows. The experimental setup is explained in section 2, the results of the OES are presented in section 3. Considering composition and transport properties of the different gas mixtures the results are discussed in section 4 .

\section{Experiment}

For the welding experiments a welding power source TPS5000 and torch from FRONIUS have been used applying the cold metal transfer (CMT) mode and a corresponding control characteristic (C1). The solid wire was made of steel (G3Si1) of a diameter of $1.0 \mathrm{~mm}$ and has been set as the anode and fed at an average rate of $3.5 \mathrm{~m} / \mathrm{min}$. The workpiece was made of steel (S355) and has been moved under the torch with the welding speed of $0.3 \mathrm{~m} / \mathrm{min}$. The torch was mounted in a fixed position perpendicular to the workpiece and in a distance of $12 \mathrm{~mm}$ between the workpiece surface and the contact tip. According to established practise, two options of the welding gas have been 
applied, namely a) Corgon $\mathrm{S} 8\left(92 \% \mathrm{Ar}+8 \% \mathrm{O}_{2}\right.$, percentage means molar fraction) and b) $100 \% \mathrm{CO}_{2}$, both with a flow rate of $12 \mathrm{slm}$. Corgon $\mathrm{S} 8$ has been chosen as a representative of a welding gas where the intert gas content dominates the properties.

The arrangement of electrical and optical diagnostics is sketched in figure 4 . The arc current has been measured with a Hall-effect transducer, whereas the voltage has been determined with a high voltage differential probe connected with the contact tip of the wire. The arc has been observed by a digital high speed camera HG $100 \mathrm{~K}$ from Redlake with a recording frequency of 20000 frames/s and exposure times of 5 to $47 \mu \mathrm{s}$. This camera is positioned at right angle to the workpiece movement.

To study the changes in the arc structure, optical emission spectroscopy has been applied focussing on the high-current phase of the arc. A $0.5 \mathrm{~m}$ spectrograph has been used with a grating $150 \mathrm{~mm}^{-1}$ combined with an intensified CCD camera from Princton Instruments. Side-on emission profiles have been recorded over the arc cross section in a distance of $1.7 \mathrm{~mm}$ from the workpiece surface, i.e., on a line perpendicular to the arc axis. The arc has been observed looking in the direction opposite to the workpiece movement to see the front of the arc which is not covered by the weldseam. The radiance measurements have been calibrated by a tungsten ribbon lamp.

\section{Results}

Figure 5 shows the typical voltage and current evolution over one pulse for the two welding gas options. The process includes a phase of high current (250 A) and voltage over $2 \mathrm{~ms}$ followed by a low-current phase with a lowered voltage. This phase ends with the short-circuit typically at 4 to $5 \mathrm{~ms}$ after the first high-voltage peak. The welding gas impact becomes obvious already from the electrical characteristic. The voltage drop between workpiece and wire contact is increased for $\mathrm{CO}_{2}$ gas in comparison with Corgon S8 over the whole range of the arc operation. In the beginning of the high-current phase the voltage difference is approximately $6 \mathrm{~V}$ and increases up to $10 \mathrm{~V}$ with respect to the different welding gases. For a first rough estimation of the electric power deposited in the arc, a comparable voltage drop in the wire of less than $1 \mathrm{~V}$ and over the electrode sheath regions of $13 \mathrm{~V}$ [10] can be assumed. Consequently, the electric power of the arc in the high-current phase (1 to $2 \mathrm{~ms}$ after first voltage maximum) increases approximately by a factor of 2 when using $\mathrm{CO}_{2}$ instead of Corgon $\mathrm{S} 8$ gas.

The typical behaviour found for the two welding gas options should be demonstrated by high-speed images and spectroscopic data at the time instants 0, 200, 400, 700, 1200, 1900, 2100 and $2500 \mu$ s after the first voltage peak, i.e. corresponding to the time scale given in figure 5. The first three instants should reflect the range of rise in current, the instants 700 to $1900 \mu \mathrm{s}$ the high-current phase and the remaining instants show the range of current fall to the lower current phase. Images from the high-speed camera obtained with an optical bandpass-filter (RA 61 for 650 to $700 \mathrm{~nm}$ ) at these time instants are shown in figure 6 for the welding Corgon $\mathrm{S} 8$ and in figure 7 for $\mathrm{CO}_{2}$. The filter and exposure time have been chosen in such a way that the arc structure including metal 
vapour and welding gas can be distinguished (the choice of the spectral range will become obvious from the discussion in the following paragraph). Different filters should be used to have a better view of the wire head, weld pool and droplet. As it can be seen in figure 6 in the high-current phase (see 400 to $1900 \mathrm{~s}$ ) a broad bell-shaped arc is established. The white coloured regions in the figure indicate an arc core with intense radiation which is quite thin in comparison with the bell-shaped region. Looking at the same time instants in figure 7, the bell-shaped arc-region is significantly smaller but the arc core of higher intensity is widened and fills almost the whole bell-shaped region. In addition, in figure 6 the weld pool at a much steeper slope of the weld seam can be seen in comparison with the more flat pool in figure 7.

Overview spectra corresponding to the line-of-sight through the arc centre are given in figure 8 for the times 200,700, 1200, and $1900 \mu \mathrm{s}$. They have been obtained by using exposure times between 10 to $50 \mu \mathrm{s}$. In the case of the Corgon S8 gas all dominant lines above $690 \mathrm{~nm}$ result from argon whereas the lines below $690 \mathrm{~nm}$ belong to iron atoms and ions. In the case of $\mathrm{CO}_{2}$ the strongest line representing plasma radiation of the welding gas is the line at $777 \mathrm{~nm}$ of atomic oxygen. It becomes obvious from the figure that the arc radiation is dominated by lines of the metal vapour in both cases. Considerable radiation of the welding gas has been observed in the beginning of the high-current phase only. There are also differences in the contribution of argon in the case of Corgon $\mathrm{S} 8$ and oxygen in the case of $\mathrm{CO}_{2}$. To evaluate these differences, the radiation of three lines will be analysed in more detail in the following part. The metal vapour radiation should be represented by the line at $516.75 \mathrm{~nm}$ (Fe I), the welding gas Corgon $\mathrm{S} 8$ by the argon atomic line at $696.54 \mathrm{~nm}$ and $\mathrm{CO}_{2}$ by the oxygen atomic line at $777.19 \mathrm{~nm}$. The radiation intensity of these lines has been determined by integrals over the corresponding measured line profiles. The considered argon line is sufficiently strong and well separated from neighbouring lines, whereas overlapping of line wings has to be taken into account in case of the other lines. Three lines with relevant intensity have been recorded arround $516 \mathrm{~nm}$, namely an atomic line at $516.75 \mathrm{~nm}$, an ionic line at $516.90 \mathrm{~nm}$ and an atomic line at $517.16 \mathrm{~nm}$. The latter line was of low intensity in all measurements, and its influence of the intensity of the $516.75 \mathrm{~nm}$ line has been neglected. The line at $516.90 \mathrm{~nm}$ showed an intensity comparable to that of the $516.75 \mathrm{~nm}$ line. Therefore, the spectral integration has been performed up to the wavelength corresponding to the minimum intensity between both line centres. This approach has been checked for single cases by fitting both lines with Lorentz profiles. Apart from cases of very low emission this comparison resulted in errors less than $4 \%$. A similar procedure has been applied to the oxygen line at $777.19 \mathrm{~nm}$ where the overlapping with the other lines of the triplet at $777.42 \mathrm{~nm}$ and at $777.54 \mathrm{~nm}$ has been corrected. Abel inversion is used to determine the emission coefficients $\varepsilon$ depending on the radial position $r$ under the assumption of a rotationally symmetric arc. Deviations from the rotational symmetry have been controlled by the comparison of different fits of the side-on radiance measurements and were sufficiently small. The resulting emission coefficients are given in figure 9 in absolute units at different time instants corresponding to the images in figures 6 and 
7. Notice, that the radiation intensity at the rising edge of the current $(400 \mu \mathrm{s})$ in the case of $\mathrm{CO}_{2}$ was too low for the determination of the emission coefficient (compare the images for $400 \mu \mathrm{s}$ in figures 6 and 7 ).

For both cases of the welding gas the emission coefficients reveal the dominance of metal vapour radiation in the arc centre, whereas the welding gas contributes to the plasma radiation mostly in the outer regions of the arc. For the gas Corgon S8 the Fe line radiation (see figure 9a) first increases with the current and is almost constant during the high-current phase. Here, the profile spreads up to a radius of approximately $0.5 \mathrm{~mm}$ with sharp gradients. In contrast to this, the Fe line radiation for the case of $\mathrm{CO}_{2}$ gas (see figure 9c) is much broader (up to $1 \mathrm{~mm}$ radius) and shows a lower gradient in the wings. The maximum intensity of the $\mathrm{Fe}$ line in the case of $\mathrm{CO}_{2}$ gas is larger than that in the case of Corgon S8 by a factor of 3. Notice, that almost all observed Fe lines show very similar temporal evolutions of the emission coefficients.

The radiation intensity of the Ar line $(696 \mathrm{~nm}$ ) (see figure 9b) is lower than that of the observed Fe line by a factor of about 10. It reaches its maximum in time during the current rise phase (around $400 \mu \mathrm{s}$ ) with a very broad profile and a spatial maximum in the arc centre. During the high-current phase the spatial maximum shifts towards the outer region of the arc (at $1200 \mu \mathrm{s}$ ) and back later on (at $1900 \mu \mathrm{s}$ ). The oxygen line radiation in the case of the $\mathrm{CO}_{2}$ gas (see figure 9d) has narrower profiles with a maximum at a radial position of about $1 \mathrm{~mm}$ in the beginning of the high-current phase. That means that atomic oxygen is present and emits radiation mostly in an outer shell of the arc. Nevertheless, it reaches one third of the maximum intensity of the Fe line.

\section{Discussion}

The radial profiles of the emission coefficients shown in the preceding section do not allow determination of the plasma temperature because of the additional dependence on the metal vapour density. However, estimations of the temperature and the metal vapour fraction can be deduced for the centre of the arc by applying additional assumptions. The emission coefficient $\varepsilon$ of the line at $516.75 \mathrm{~nm}$ (resulting from the transition from the upper energy level $3.88 \mathrm{eV}$ of the Fe atom) can be calculated as a function of the plasma temperature for pure iron vapour under the assumptions of local thermodynamic equilibrium (LTE) and optical thin radiation. The maximum intensity of $2.05 \times 10^{7} \mathrm{Wm}^{-3} \mathrm{sr}^{-1}$ (norm maximum) arises at approximately $8000 \mathrm{~K}$. This intensity is reduced down to nearly $75 \%$ of the maximum value at about $6800 \mathrm{~K}$ and at the higher value of $9500 \mathrm{~K}$. The emission coefficient obtained for the case of $\mathrm{CO}_{2}$ is near to this value of $75 \%$ of the norm maximum in the arc centre (see for example the instant $1200 \mu \mathrm{s})$. Hence, in the limiting case of pure iron vapour the arc centre temperature must be in the range between 6800 and $9500 \mathrm{~K}$. More probable is the situation that the arc in case of $\mathrm{CO}_{2}$ contains approximately $75 \%$ iron (molar fraction) and has a temperature of about $8000 \mathrm{~K}$ in the centre. Note that the emission intensity measured in the centre can not be generated with any lower iron content. By analogy, an iron 
fraction of about $25 \%$ (as a lower limit) and a temperature near to $8000 \mathrm{~K}$ can be estimated in the arc centre for the case of Corgon S8. In these rough estimations the small changes of the temperature dependence of the radiator partial density (neutral iron atoms) on the different gas mixtures have been neglected.

Additional conclusions can be drawn from a simple consideration of the current transfer through the arc cross section. Therefore, the arc length has been approximately determined from the figures 6 and 7). The high-current phase at the time $1200 \mu \mathrm{s}$ is considered as a typical example for both cases of the welding gas. The arc lengths $2.4 \mathrm{~mm}$ for Corgon S8 and $2.0 \mathrm{~mm}$ for $\mathrm{CO}_{2}$ have been obtained. As lower and upper limits of the voltage drop in the wire and over the electrode sheath regions $8 \mathrm{~V}$ and $14 \mathrm{~V}$ are assumed. Measured voltages of $24 \mathrm{~V}$ and $32 \mathrm{~V}$, respectively, result in electric field strengths between 42 and $67 \mathrm{~V} / \mathrm{cm}$ for Corgon S8 and between 90 and $120 \mathrm{~V} / \mathrm{cm}$ for $\mathrm{CO}_{2}$ assuming an axially homogeneous arc column. In the following, the hypothesis should be investigated, that the arc core region dominated by the metal vapour radiation carries the whole arc current. As a rough approach, this core region spreads until the radial position, where the iron emission coefficient is reduced to half of its maximum. This gives $0.57 \mathrm{~mm}$ radius for the case of Corgon $\mathrm{S} 8$ and $0.97 \mathrm{~mm}$ and $8.5 \mathrm{kA} / \mathrm{cm}^{2}$ for $\mathrm{CO}_{2}$. If these radii would be equal to the effective radii of the current carrying channel, then current densities of $24.5 \mathrm{kA} / \mathrm{cm}^{2}$ for the case of Corgon $\mathrm{S} 8$ and $8.5 \mathrm{kA} / \mathrm{cm}^{2}$ for $\mathrm{CO}_{2}$ corresponding to the identical current of $250 \mathrm{~A}$ are obtained. In case of Corgon S8 an electrical conductivity between 3.6 and $59 \times 10^{4} \mathrm{AV}^{-1} \mathrm{~m}^{-1}$ results from the hypothesis. The analogous considerations for the case of $\mathrm{CO}_{2}$ lead to an electrical conductivity between 7 and $9.4 \times 10^{3} \mathrm{AV}^{-1} \mathrm{~m}^{-1}$.

For the following discussion, the dependence of the relevant thermodynamic and transport properties of the arc on the temperature and the gas composition is considered. Figure 10 shows the electrical and thermal conductivity as well as the specific enthalpy of the plasma considering different mixture compositions of iron vapour and welding gas for temperatures from 3000 up to $9500 \mathrm{~K}$. These values have been calculated together with the plasma composition by standard methods (minimisation of the free Gibbs energy, Chapman-Enskog method for the solution of the kinetic equation) and considering LTE [11]. Ignoring possible deviations from the LTE, these values reflect the range of properties of the welding arcs under observation.

From figure 10a it becomes obvious that electrical conductivities estimated for the case of Corgon S8 in the preceding paragraph can hardly be realised in the plasma column for any gas composition. Consequently, the effective radius of the current path must be considerably larger than the estimated radius of the region of iron vapour radiation in case of Corgon S8. In case of $\mathrm{CO}_{2}$, temperatures above $10000 \mathrm{~K}$ are necessary to reach the above estimated conductivity which contradicts the temperature values estimated from the iron emission in the arc centre. Considering arc centre temperatures around $8000 \mathrm{~K}$ and iron molar fractions in the range between 25 and $95 \%$, as estimated above, an electrical conductivity in the range between 3 and $5 \times 10^{3} \mathrm{AV}^{-1} \mathrm{~m}^{-1}$ can be expected. 
To get the order of magnitude of the effective radius of the current carrying path, a mean electrical conductivity of $4 \times 10^{3} \mathrm{AV}^{-1} \mathrm{~m}^{-1}$ can be considered for simplicity (see the thin horizontal line in figure 10a). This, together with the estimations of the electric field in the arc, leads to an effective radius between 1.7 and $2.2 \mathrm{~mm}$ for the case of Corgon S8 and between 1.3 and $1.5 \mathrm{~mm}$ for the case of $\mathrm{CO}_{2}$ in the high-current phase (instant $1200 \mu \mathrm{s}$ for example). The arc current of $250 \mathrm{~A}$ causes mean current densities between 1.6 and $2.7 \mathrm{kA} / \mathrm{cm}^{2}$ for Corgon $\mathrm{S} 8$ and between 3.5 and $4.8 \mathrm{kA} / \mathrm{cm}^{2}$ for $\mathrm{CO}_{2}$, i.e., nearly twice of the density for Corgon S8. Note, that these estimations refer to the cross section of the arc at the observation plane $1.7 \mathrm{~mm}$ above the weld pool.

Three conclusions can be drawn from these estimations, firstly the current transfer occurs not only in the region of large metal vapour emission but also in regions with low metal content dominated by the welding gas. Secondly, this effect is more pronounced in the case of Corgon S8, where the current path must be much broader than the region of the iron emission and also broader than the current path for the case of $\mathrm{CO}_{2}$. As the third conclusion, temperatures higher than $5000 \mathrm{~K}$ must be expected in the regions of the larger emission of the welding gas components. Otherwise these regions would not be able to contribute to the current transfer. In addition, this regions can contain only very small amounts of metal vapour because of the low iron emission.

The very different radial structure of the arcs in the cases of the two welding gases deduced in the preceding discussion can be interpreted by considering the heat transfer in radial direction. Expecting the temperature maximum as well as the maximum of the Ohmic heating in the centre of the arc, the energy transport from inner to outer regions will contribute to a radial enlargement of the hotter regions of the arc. A mixture with high content of $\mathrm{CO}_{2}$ has the lowest thermal conductivity at least above $4000 \mathrm{~K}$, and the highest specific enthalpy above $6000 \mathrm{~K}$ in comparison with the other mixtures under consideration, as can be seen from figure 10. Hence, the $\mathrm{CO}_{2}$ gas can cool the arc in the outer region very efficiently and may generate a strong temperature gradient. This effect has been demonstrated among others by computational studies [12] and has been named "thermal pinch effect" [13].

Argon, as well as mixtures with a large molar fraction of Ar, have a significant lower specific heat in the whole temperature region, resulting in a less efficient cooling of the arc edge. In addition, the higher thermal conductivity in the range between 4000 and $6000 \mathrm{~K}$ supports the energy transport to the arc surroundings and, hence, a broadening of the hotter region. This transport as well as the considerable Ohmic heating connected with the current transfer outside of the metal vapour core supports the enlargement of the region of considerable argon emission in case of Corgon S8.

The more constricted arc corresponding to the estimations of the current path dimensions in case of $\mathrm{CO}_{2}$ obviously leads to a smaller arc root at the wire which may support higher temperatures of the droplet surface and, consequently, a higher metal evaporation rate. This corresponds to a higher iron molar fraction in the arc centre estimated for this case, which is required for a sufficient high electrical conductivity in comparison with pure $\mathrm{CO}_{2}$ gas at the same temperature. 
To summarise, the interpretation of the radiation profiles indicate a stronger temperature gradient in the outer region of the arc in the case of $\mathrm{CO}_{2}$ as the welding gas. In contrast, argon supports a broader arc profile and an energy balance at a lower level caused by the lower current density. Furthermore, it is assumed that the radial profiles of the arc properties, which have been analysed at $1.4 \mathrm{~mm}$ away from the weld pool, continue up to the weld pool surface. In such a case, more energy in a more focussed region should be transmitted to the work piece in the case of $\mathrm{CO}_{2}$. The energy transfer should be lower and distributed over a larger region in the case of Corgon S8.

\section{Summary and conclusion}

The arc in the high-current phase of a CMT process has been investigated for the welding gases Corgon $\mathrm{S} 8$ and $\mathrm{CO}_{2}$. Radial profiles of the emission coefficients of iron vapour as well as of the welding gases represented by argon and oxygen, respectively, have been determined from side-on spectra along the cross section of the arc in a distance of $1.7 \mathrm{~mm}$ from the workpiece. The large iron line emission (near to the norm maximum of the considered line in case of $\mathrm{CO}_{2}$ ) indicates the strong vaporisation, and the arc core is mainly determined by the iron vapour properties. The welding gas radiation reaches its maximum in the outer region of the arc. Among plausible assumptions a consistent interpretation has been obtained explaining the different effects of the welding gases, a molecular gas with lower electrical conductivity or an inert gas with lower specific heat. In particular, rough estimates of the temperature and the iron molar fraction in the arc centre as well as of the dimension of the current path and, hence, the mean current density in the observation plane could be deduced for both cases of the welding gas.

The quantitative validation and more detailed proof requires the determination of densities of plasma species and plasma temperature either by modeling or by high-

resolution spectroscopy. The latter was out of the scope of the present paper in view of the rapid variation in time of the CMT process and is aspired in future work.

\section{Acknowledgment}

The authors want to thank Ms. Francesca Cassidy for fruitful discussions of the paper.

\section{References}

[1] Silva R H, Dutra J C and Gohr R 2008 Soldagem \& Inspecao 13 70-81

[2] Feng J C, Zhang H T and He P 2009 Materials and Design 30 1850-1852

[3] Zhang H T, Feng J C, He P, Zhang B B, Chen J M and Wang L 2009 Mat. Sci. Engineer. A Struct. Mat. Porp. Microstruct. Process. 499 111-113

[4] Wiese W L 1991 Spectrochim Acta B - Atom Spectrosc. 46 831-841

[5] Ton H 1975 Physical properties of the plasma-MIG welding arc, J. Phys. D: Appl. Phys. 8 922-933.

[6] Lenivkin V A, Varukha E N, Petrov P I, Dyurgerov N G, Gufan R M and Mergold V M 1981 Weld. Prod. 11 9-11 
[7] Mirapeix J, Cobo A, Conde O M, Jauregui C and Lopez-Higuera J M 2006 Optical Engineering, 45

[8] Alfaro S C A, Mendonca D D and Matos M S 2006 J Mat Process Technol, 179 219-224

[9] Zielinska S, Musiol K, Dzierzega K, Pellerin S, Valensi F, de Izarra C and Briand F 2007 Plasma Sources Sci. Technol. 16 832-838

[10] Hajossy R and Morva I 1994 J. Phys. D: Appl. Phys. 27 2095-2101

[11] CEA - Chemical Equilibrium with Applications, NASA Computer Programm, Glenn Research Center

[12] Tanaka M and Lowke JJ 2007 J. Phys. D: Appl. Phys. 40 R1-R23

[13] Murphy A B, Tanaka M, Tashiro S, Sato T and Lowke J J 2009 J. Phys. D: Appl. Phys. 42 115205 


\section{List of figures}

Figure 1: Sketch of current and voltage vs. time for the short circuit transfer process.

Figure 2: Spatter generation during the standard short circuiting metal transfer.

Figure 3: Sketch of current and voltage vs. time (upper part) and high speed images of the different process phases (lower part) for a typical CMT process with a wire diameter of $1.2 \mathrm{~mm}$.

Figure 4: Setup for the optical diagnostic of the welding arc.

Figure 5: Evolution of current $I$ and voltage $U$ during a typical pulse period of the CMT process for the welding gases Corgon $\mathrm{S} 8$ (upper part) and $\mathrm{CO}_{2}$ (lower part). The dashed lines denote times of the spectroscopic observations.

Figure 6: Arc images during the impulse phase when using Corgon S8 as welding gas

Figure 7: Arc images during the impulse phase when using $\mathrm{CO}_{2}$ as welding gas

Figure 8: Overview spectra from a line-of-sight through the arc centre at different times in the pulse period for the welding gases Corgon S8 (upper part) and $\mathrm{CO}_{2}$ (lower part).

Figure 9: Radial profiles of the emission coefficient $\varepsilon$ a) of the $516 \mathrm{~nm}$ line of Fe I for Corgon S8, b) of the $696 \mathrm{~nm}$ line of Ar I for Corgon S8, c) of the $516 \mathrm{~nm}$ line of $\mathrm{Fe} \mathrm{I}$ for $\mathrm{CO}_{2}$, and d) of the $777 \mathrm{~nm}$ line of $\mathrm{O} \mathrm{I}$ for $\mathrm{CO}_{2}$.

Figure 10: Electrical conductivity $\sigma$ (a), thermal conductivity $\lambda(\mathrm{b})$ and specific enthalpy $h_{0}$ (c) of different mixtures of iron vapour and Corgon S8 (Ar + $\left.8 \% \mathrm{O}_{2}\right)$ or $\mathrm{CO}_{2}$ as functions of temperature assuming LTE. The percentage values in the legend correspond to the molar fractions. 


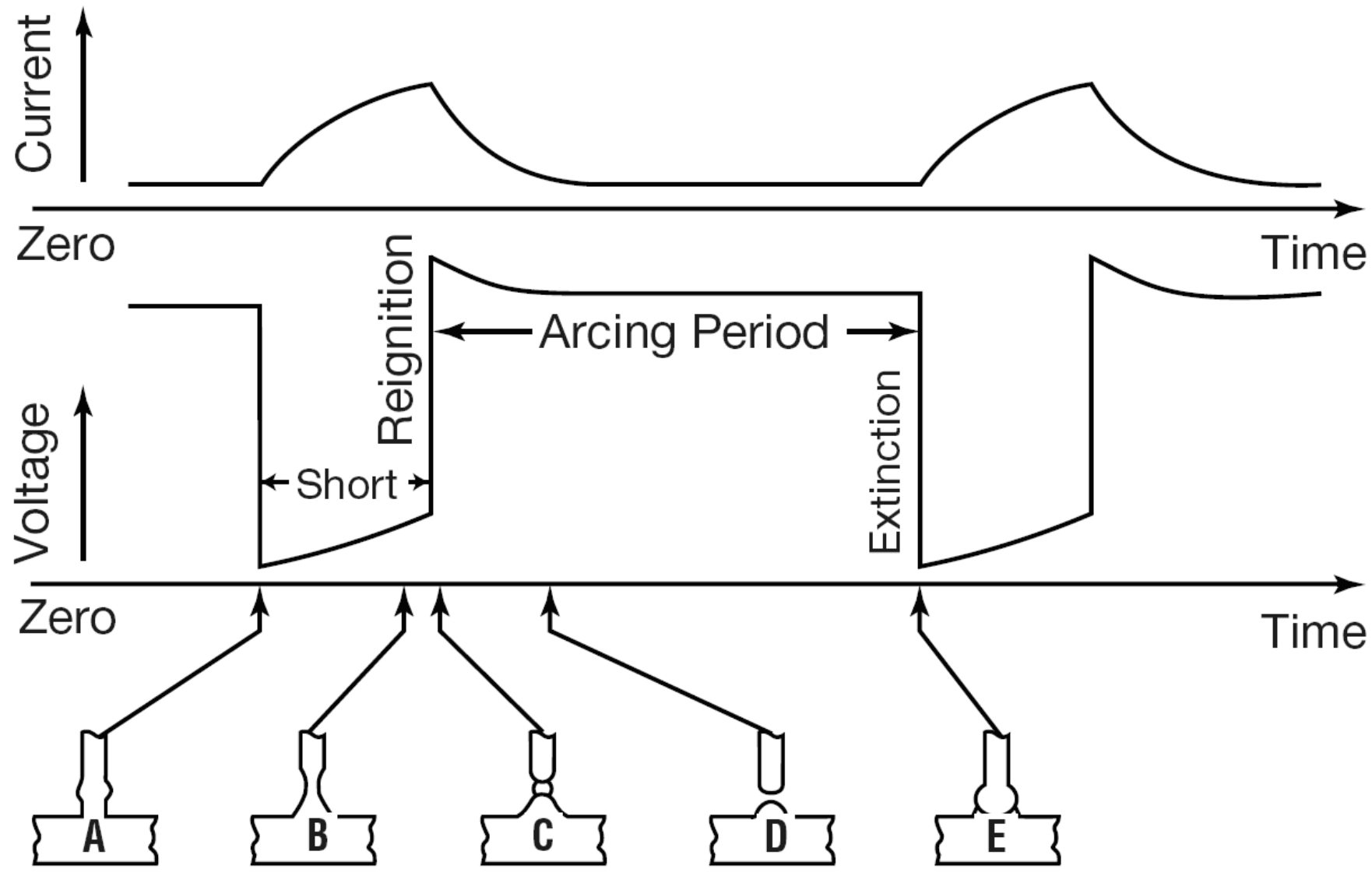

Figure 01 (Fig01.png) 


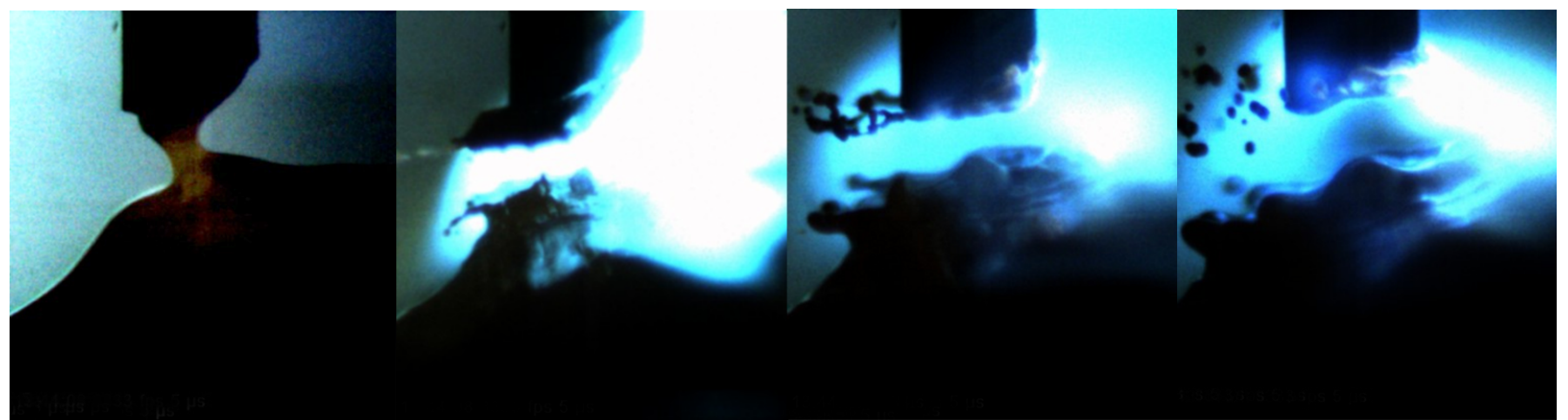

Figure 02 (Fig02.png) 


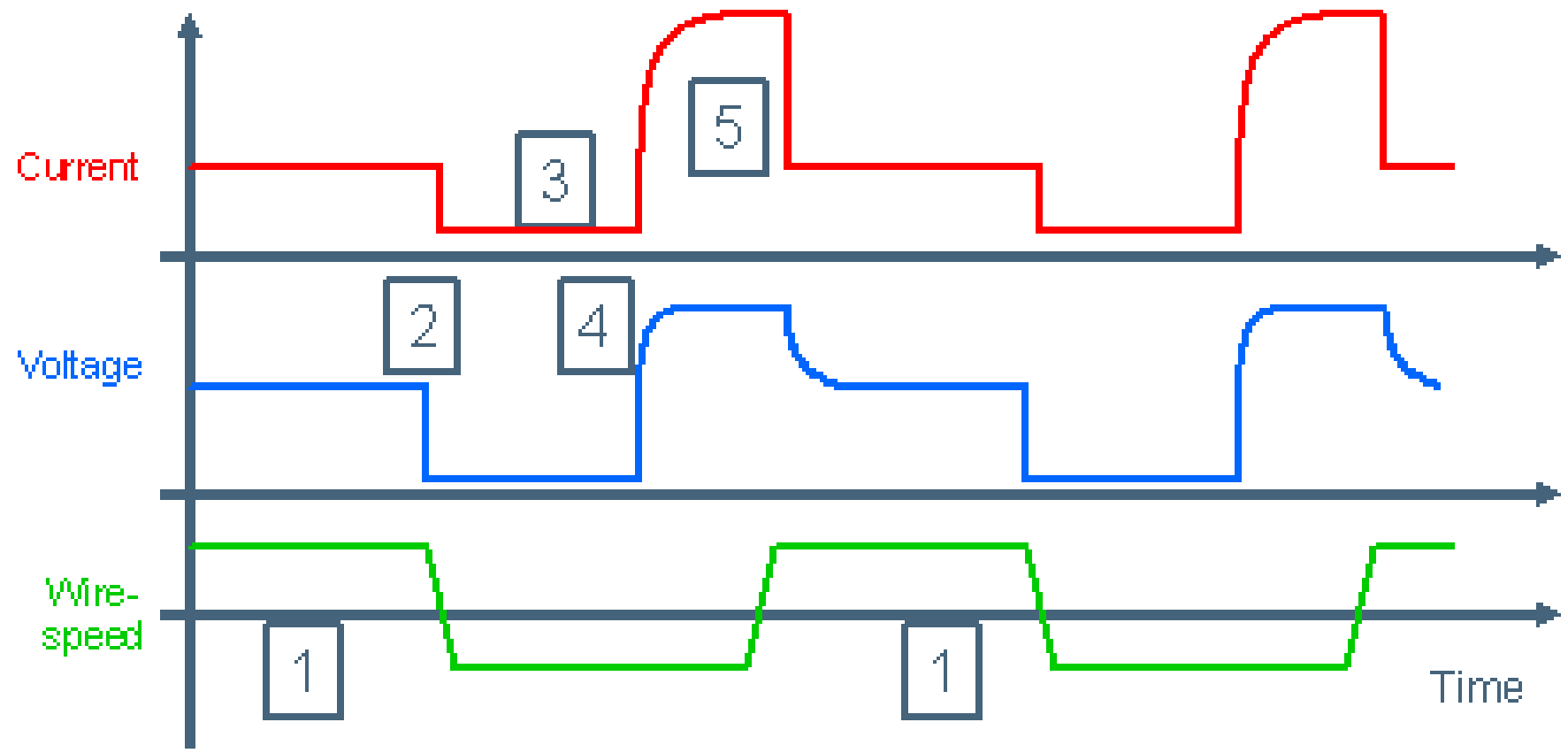

Figure 03a (Fig03a.png) 

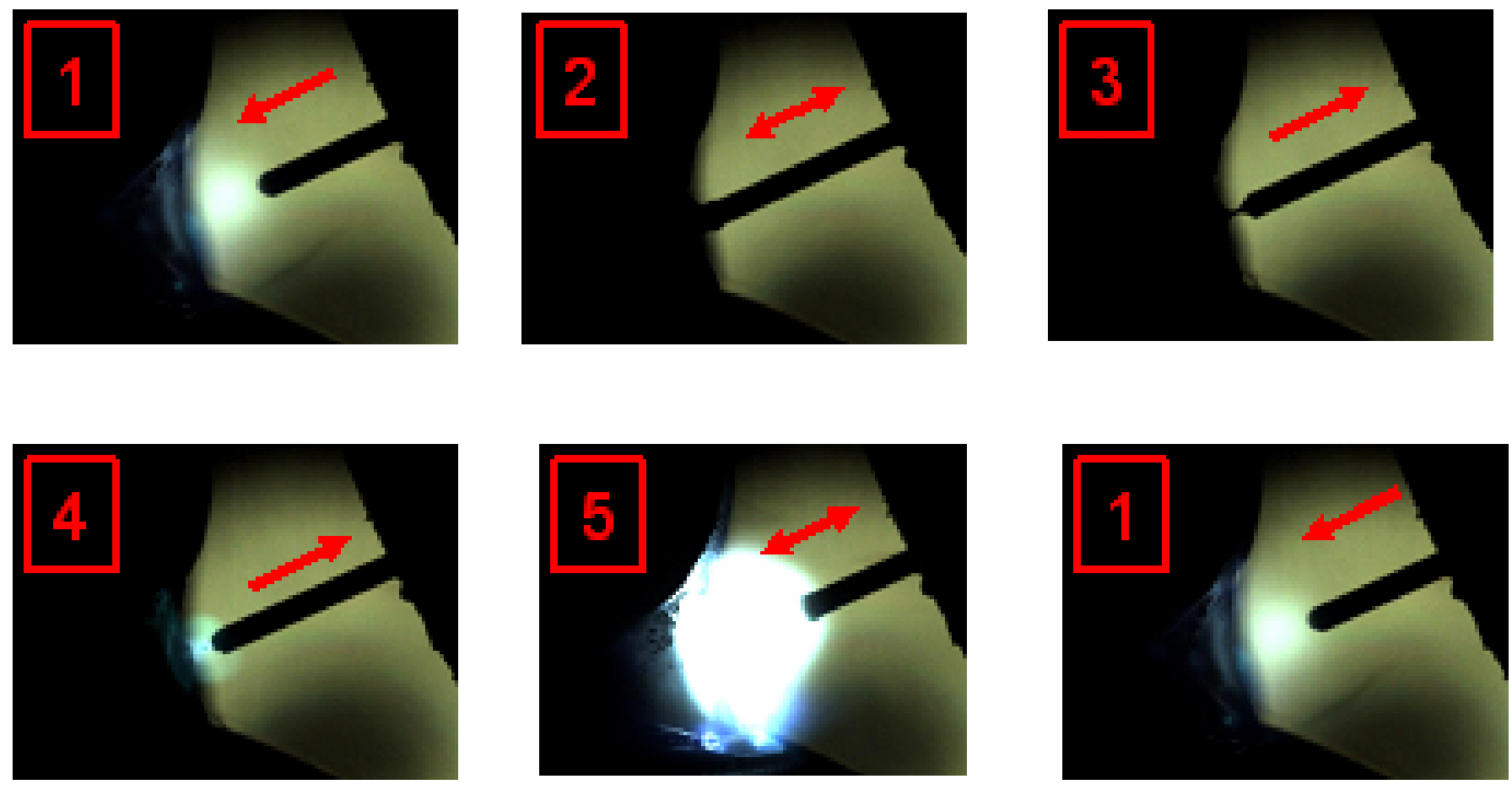

Figure 03b (Fig03b.png) 


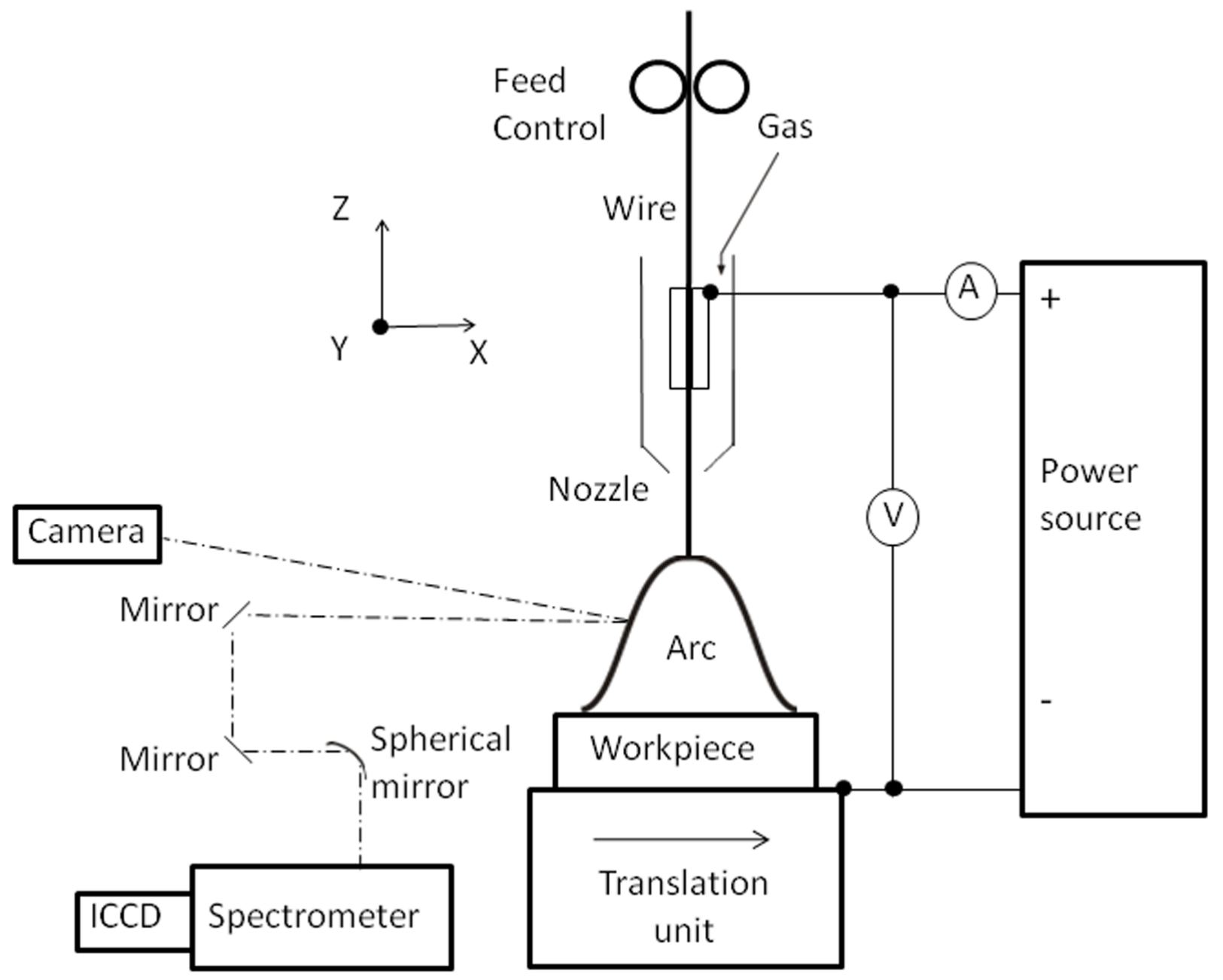

Figure 04 (Fig04.png) 


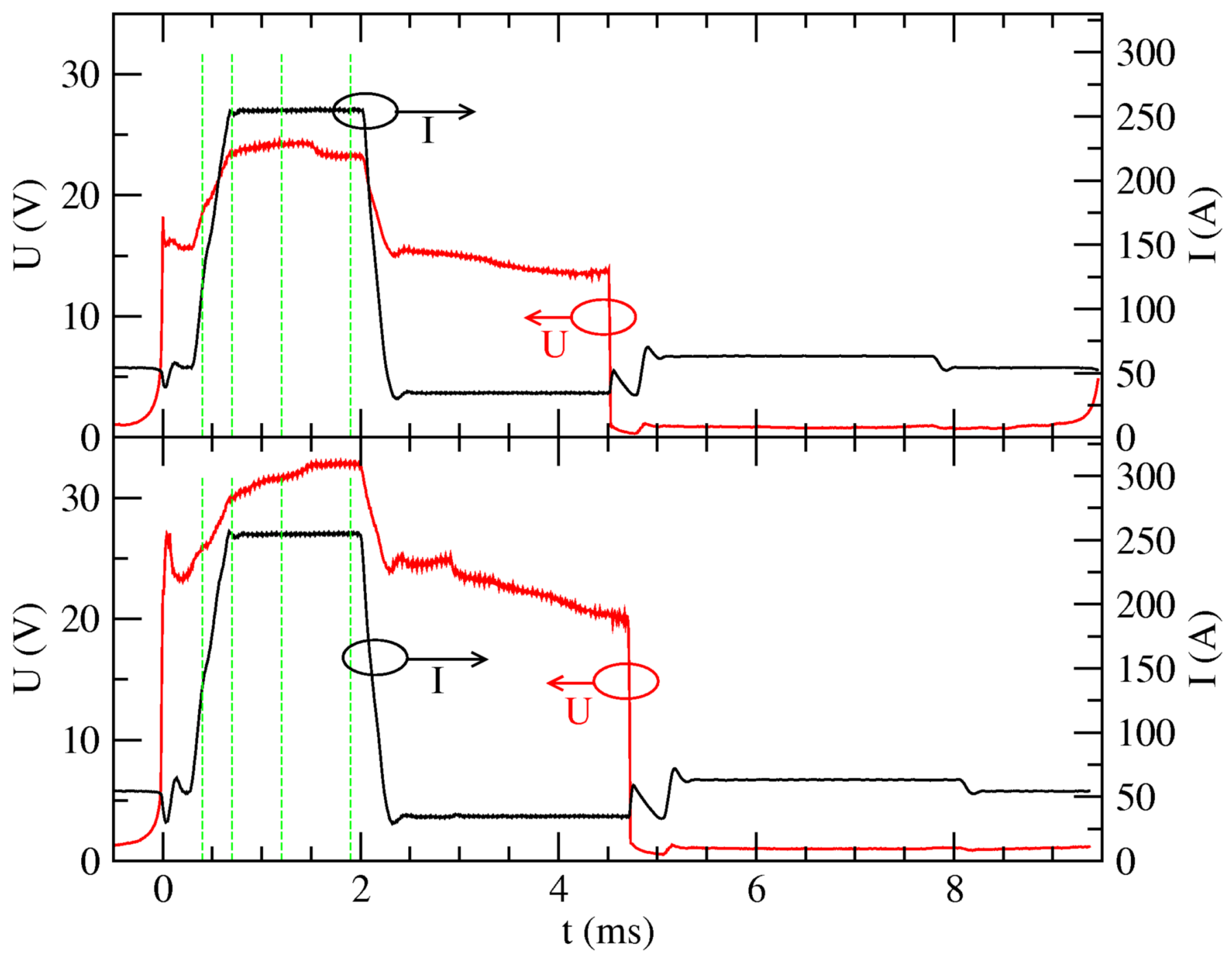

Figure 05 (Fig05.png) 

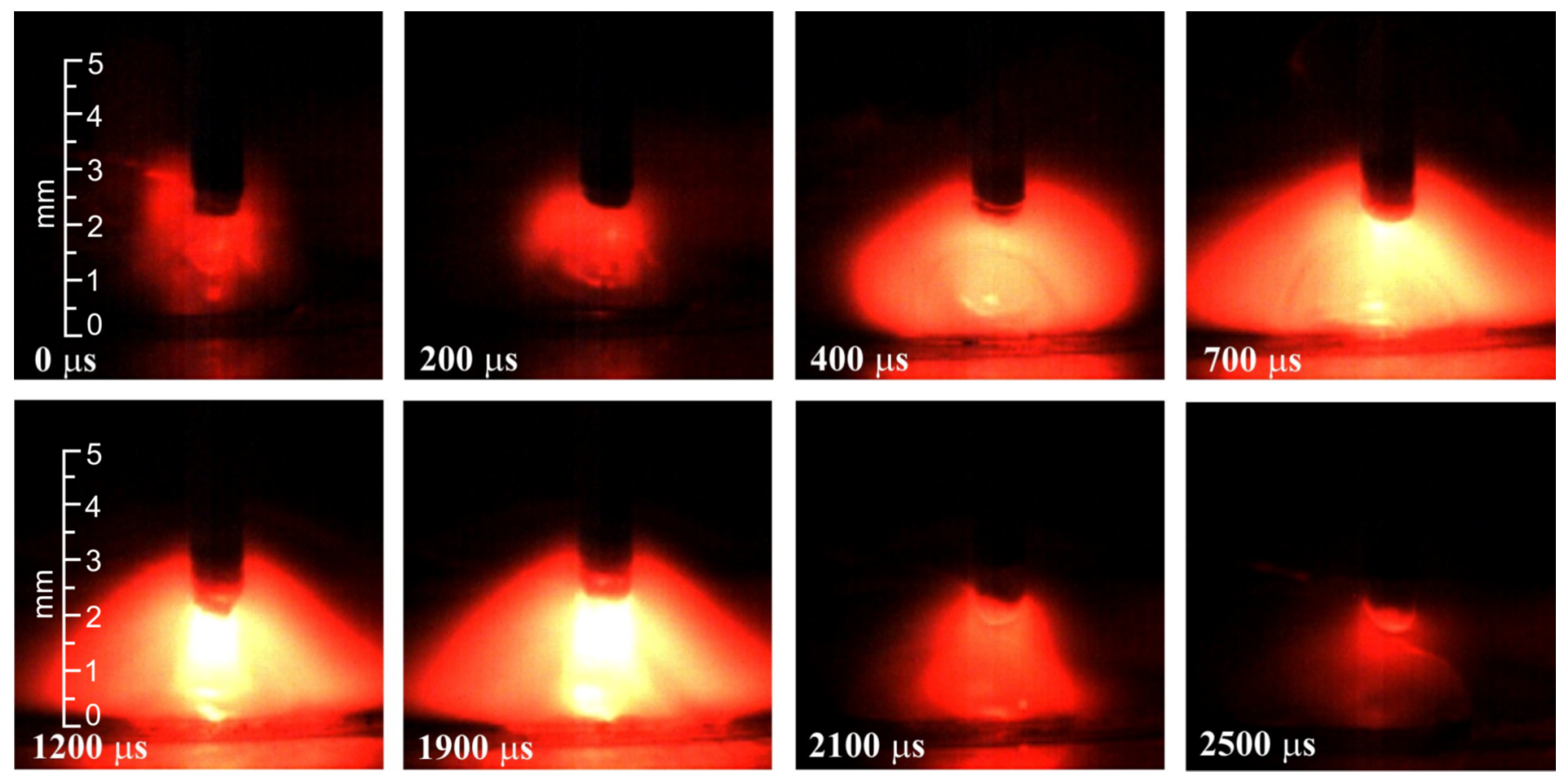

Figure 06 (Fig06.png) 

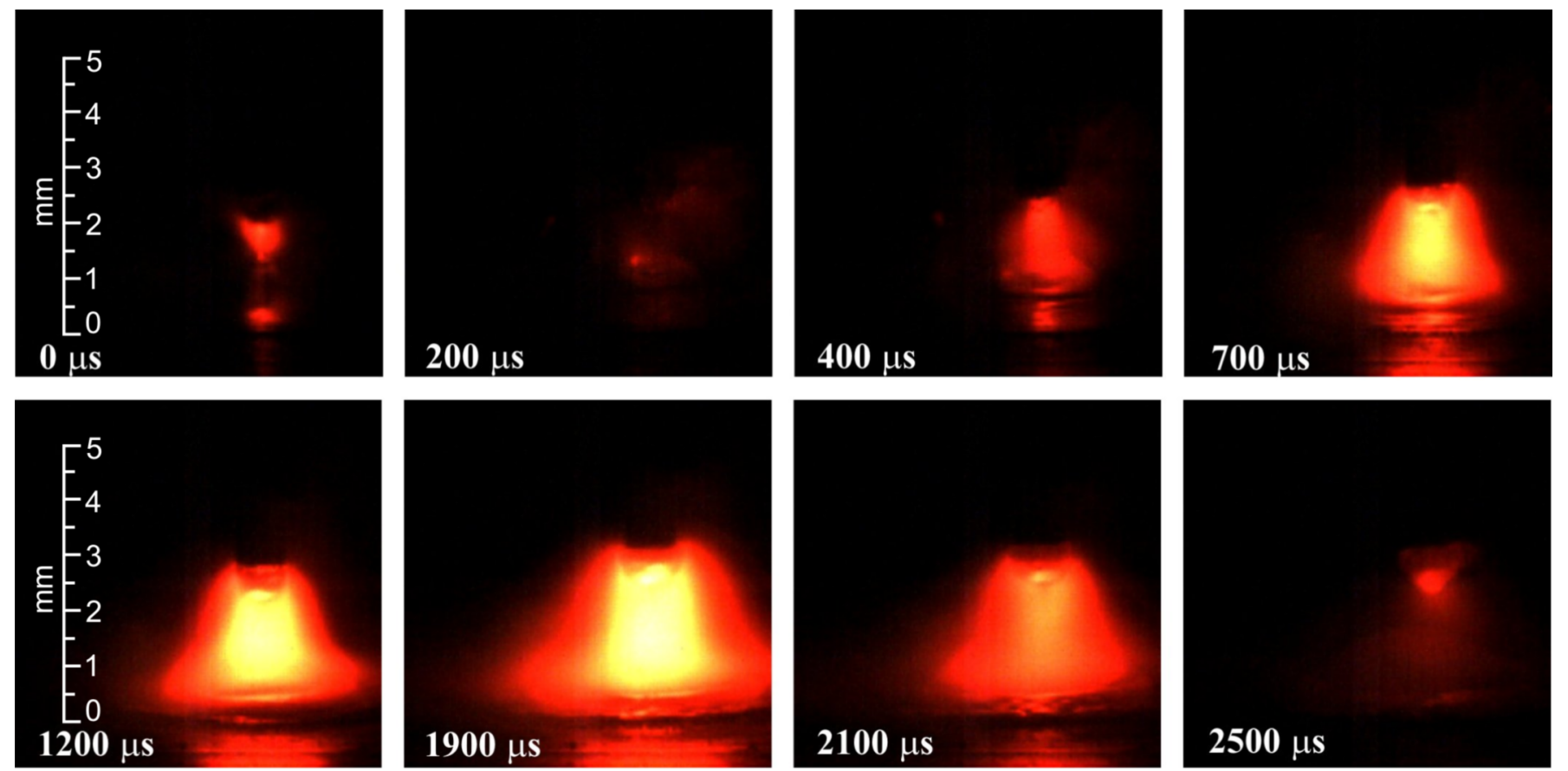

Figure 07 (Fig07.png) 


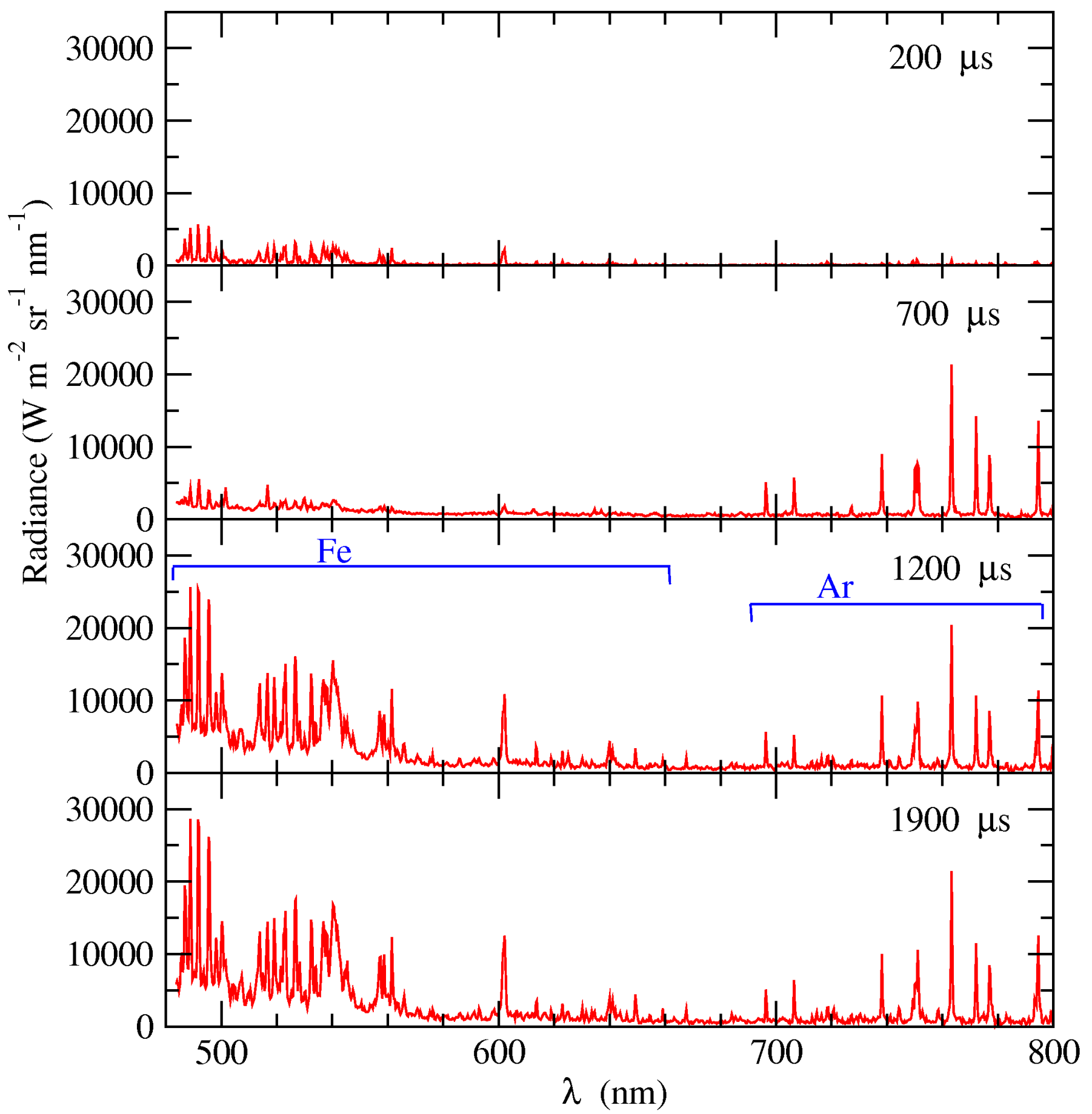

Figure 08a (Fig08a.png) 


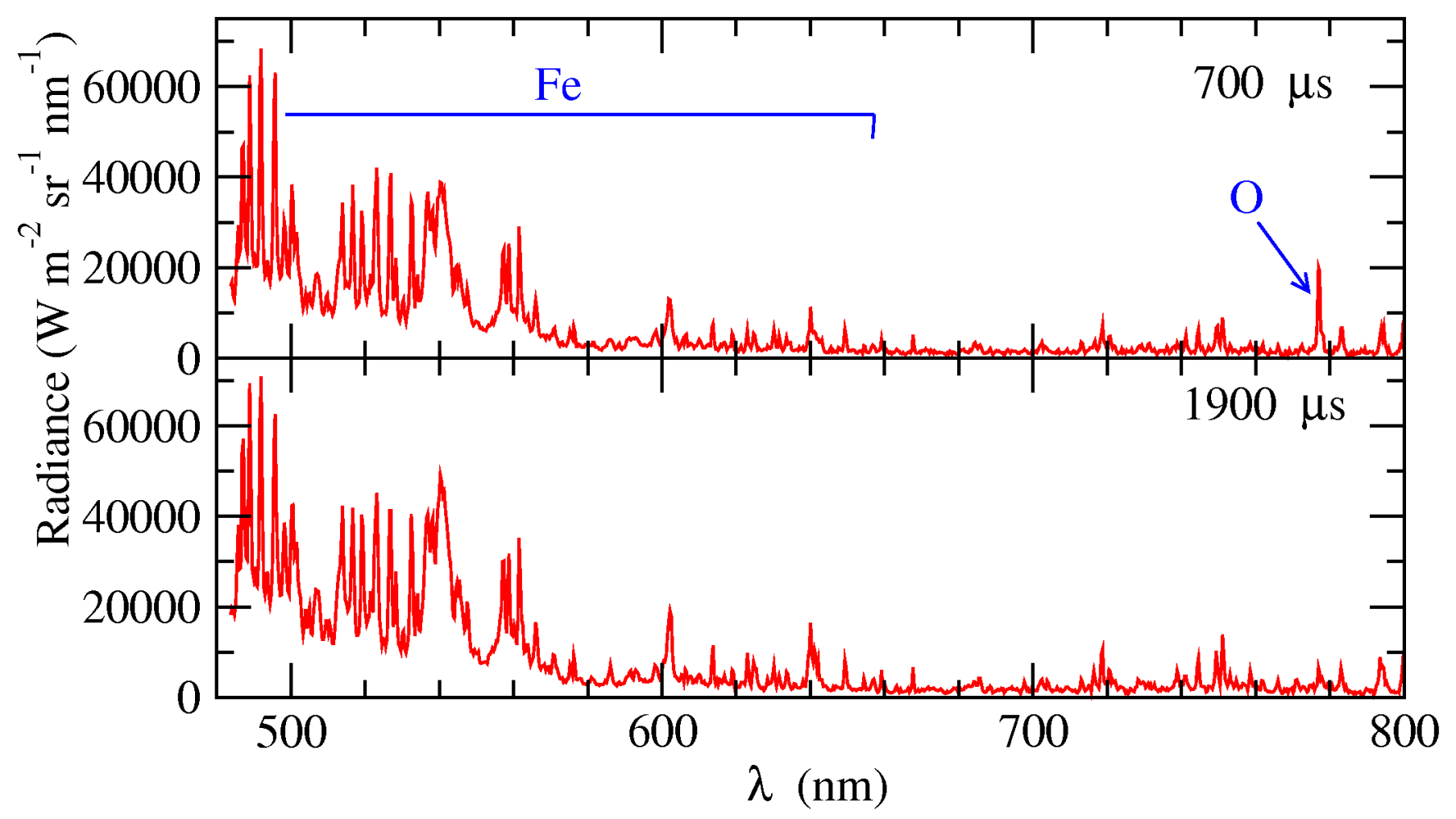

Figure 08b (Fig08b.png) 


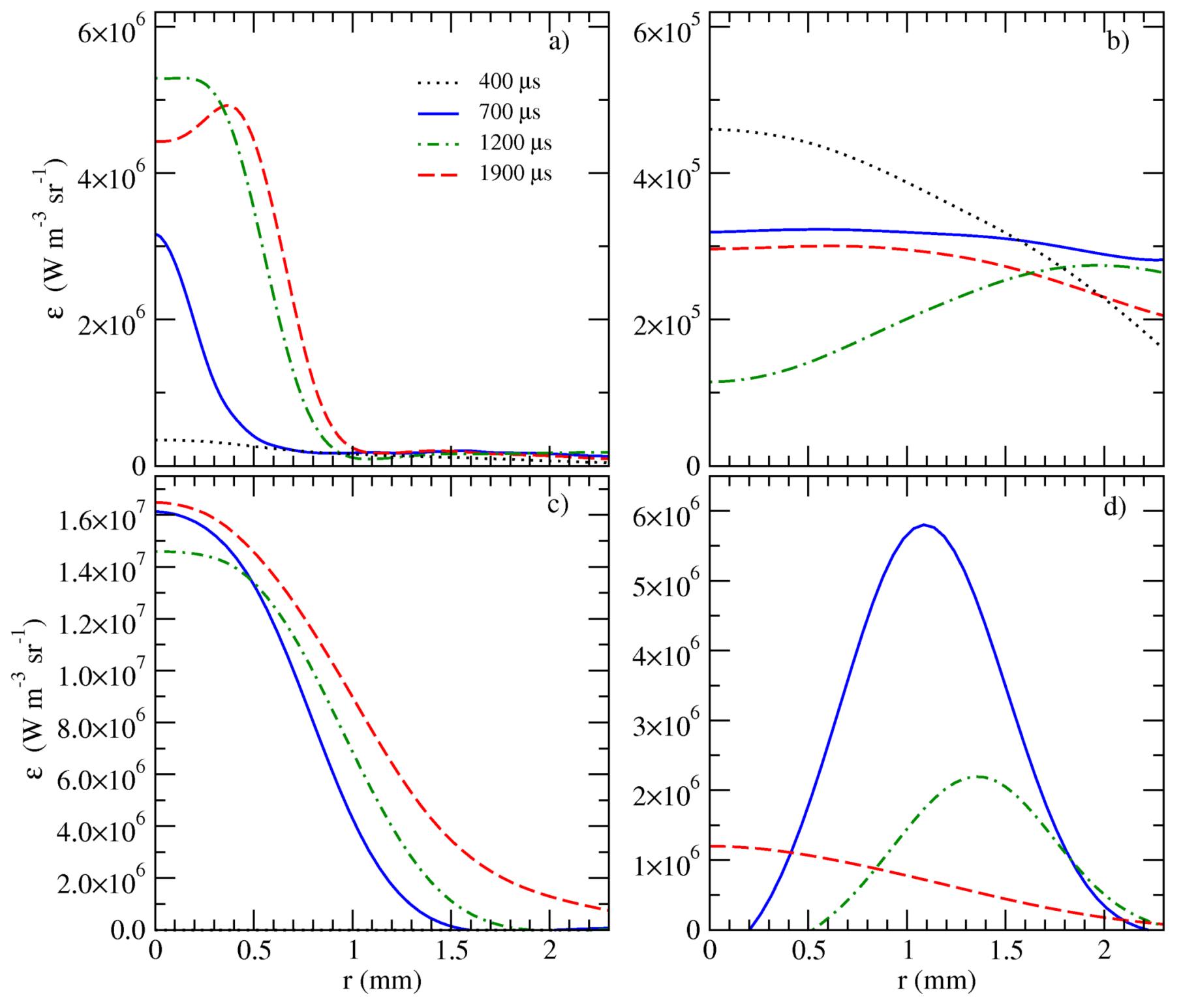

Figure 09 (Fig09.png) 


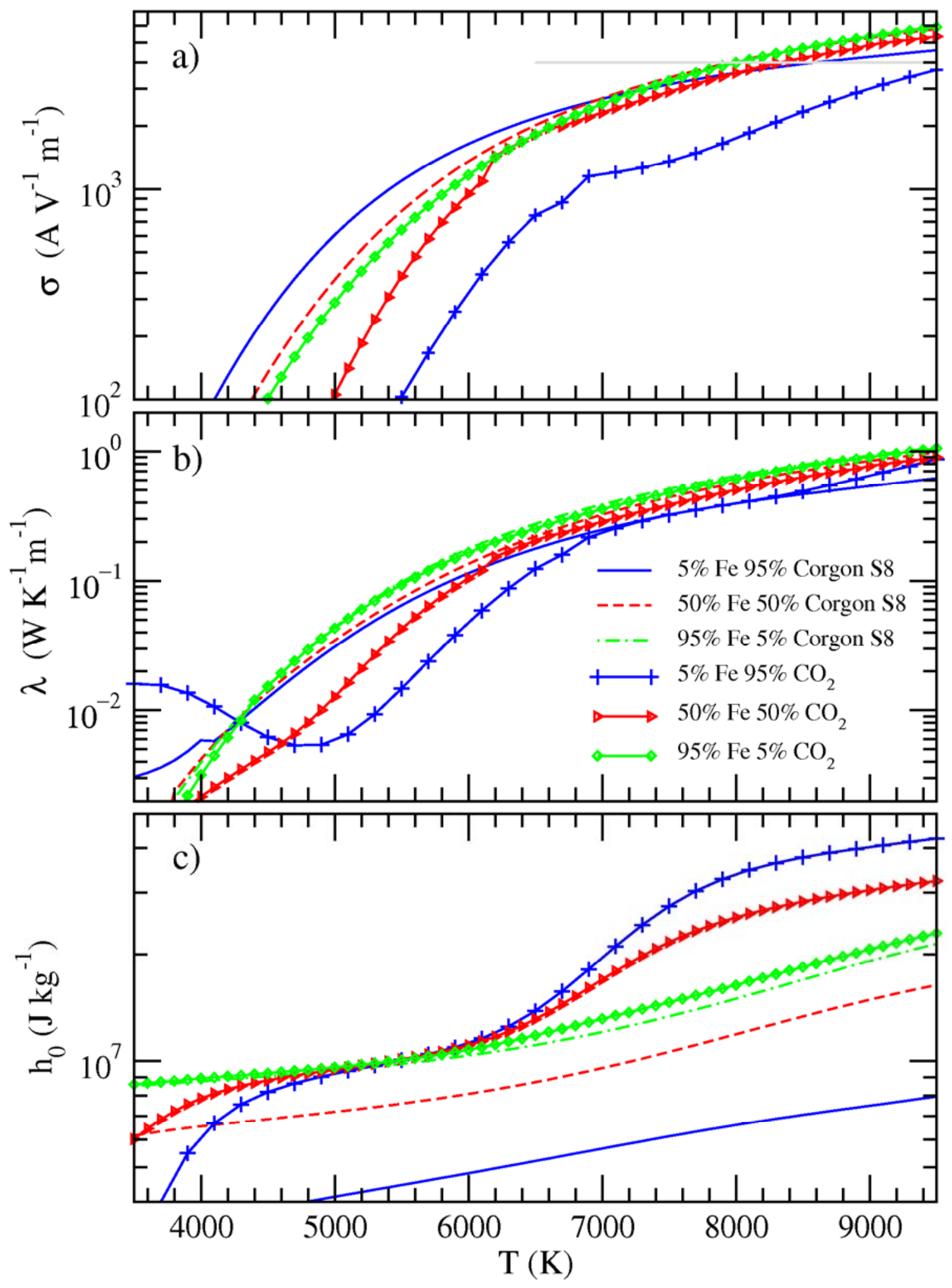

